\title{
Forma de gobernanza frente a la gestión del riesgo. Estudio de caso sector de Nueva Esperanza, Bogotá D.C., Colombia
}

\author{
Governanceform facing risk management. Casestudy \\ of the "Nueva Esperanza" sector in Bogotá, D. C., \\ Colombia
}

\section{Alexandra Bedoya Prado* Jorge Ruiz**}

\section{Resumen}

Se presentan los principales aspectos de un estudio de caso en el sector de Nueva Esperanza, localidad Rafael Uribe Uribe, Bogotá, D. C. en donde se dio una ocupación ilegal de terrenos inestables intervenidos por sus pobladores. Afectado por un fuerte deterioro ambiental y un proceso crítico de remoción en masa, la situación ha obligado en los últimos cuatro años, a adelantar una gestión del riesgo buscando mayor cooperación entre actores públicos y privados. La pregunta que se aborda es: ¿Cuál ha sido la forma de gobernanza frente a la gestión del riesgo y cómo se reflejan sus resultados en la organización espacial y el ordenamiento territorial en Nueva Esperanza? Para responderla, se identifican las causas del problema y, aplicando el M arco Analítico de Gobernanza, se aborda la caracterización de actores, la revisión de normas, la identificación de puntos nodales o de relacionamiento entre los actores, y se hace seguimiento al proceso de gestión del riesgo, analizando los efectos en la organización espacial. Se evidenció descoordinación entre actores, poca credibilidad en las entidades y un proceso de ocupación sin planificación, con aciertos y desaciertos que arrojan elementos de análisis para comprender una gobernanza de la gestión del riesgo. 
Palabras clave: Bogotá, D.C.; Gestión de riesgos; Deslizamiento; gobernanza; ordenamiento territorial; riesgos naturales.

\section{Abstract}

The "Nueva Esperanza" sector, located in the Rafael Uribe Uribe borough of Bogotá, D. C. was subject to an illegal occupation of unstable land. Affected by strong environmental degradation and critical mass removal, the situation has forced in the last years to pursue a risk management that entails greater cooperation among public and private actors. The question that this paper addresses is: Which has been the form of governance in light of risk management and how is this reflected in spatial organization and land planning? In order to encounter an answer, the causes of the problem are identified applying the governance analytical framework, actor characterization, norm revision, nodal point identification or actor relations, risk management follow-up, analyzing the effects on spatial organization. Lack of coordination among actors was detected, poor credibility of institutions was found and an occupation process without plans, with achievements and downfalls, that bestow elements of analysis in order to comprehend one governance of risk management .

Key words: Bogotá, D.C.; Governance; Landslide; land use planning, natural risks; risk management. 


\section{Introducción}

La producción social del espacio se fundamenta en una relación constante de los seres humanos con el medio natural, por medio de la ocupación, uso y transformación de dicho medio, con el fin de adecuarlo para su aprovechamiento. En ocasiones, la forma de intervención ha generado riesgos ambientales. En Bogotá, D.C., la evidencia de riesgos ambientales, como parte de una dinámica social, se observa en casos como Nueva Esperanza, Altos de La Estancia, Arborizadora Alta (localidad de Ciudad Bolívar) y El Codito (localidad de Usaquén), entre otros $\mathrm{y}$, en ciertos casos, de la mano con el surgimiento de ocupaciones ilegales carentes de servicios públicos, vías de acceso adecuadas y población en alto grado de vulnerabilidad social (CVP, 2005).

Este documento aborda el caso de Nueva Esperanza como un problema geográfico-ambiental y de ordenamiento territorial, cuyo análisis se fundamenta en cuatro ejes conceptuales: espacio geográfico, ordenamiento territorial, gestión del riesgo y gobernanza.

\section{Marco Teórico}

El espacio geográfico, como producto de la acción humana, es UN PRODUCTO SOCIAL, que se construye y se transforma a partir de una interrelación entre procesos naturales y sociales, indisolubles y mutuamente "incluyentes", en donde coexisten procesos bajo una relación entre sociedad y naturaleza. Se requiere su estudio integral para la comprensión y análisis de los procesos de su configuración, teniendo en cuenta su proceso históricogeográfico (Santos, 2000; Harvey, 1977; Gould, 1996; Ortega Varcárcel, 2000).

Massiris (2003) sostiene que tanto el espacio geográfico, como las acciones humanas que se dan en él, responden a principios y prácticas que permiten comprender mejor la organización espacial. Plantea, además, la estrecha relación entre la teoría geográfica y las acciones de ordenamiento territorial, en donde es central la idea de la relación sociedad-naturaleza, a través del tiempo.

Esta relación se hace visible en el ordenamiento territorial, entendido como un proceso e instrumento de planificación que busca regular, orientar y proyectar, en el largo plazo, la ocupación y uso del espacio geográfico, bajo un carácter técnico, político y administrativo, acorde con las potencialidades y limitaciones del mismo, el uso sustentable de los recursos y teniendo en cuenta las necesidades e intereses de la población, con el fin de mejorar su calidad de vida (Massiris, 2003; Borja, 2000; Gómez, 2002; Zoido, 1998; Morcillo, 2002;).

Los riesgos y la ocurrencia de desastres surgen, en muchas ocasiones, en el contexto de la apropiación social del espacio geográfico, dándole sentido a una de las tendencias teóricas que 
definen los riesgos como unproducto social, porque emana de la relación sociedad-naturaleza, en el proceso de construcción de las ciudades (Ramírez, 1996; Mansilla, 2000) y en su contexto histórico, como resultado de procesos sociales, políticos y económicos (García, 1996). Las dinámicas implícitas entre lo natural y lo humano han generado desequilibrios que, en ocasiones, conducen a desastres, impactan el espacio geográfico y generan cambios en la política orientada hacia el ordenamiento territorial.

El riesgo es la probabilidad de que ocurra daño a una población y su infraestructura, con efectos económicos, sociales, ambientales y políticos adversos, como consecuencia de su exposición a una o varias amenazas. Resulta de la coexistencia de la amenaza (fenómenos) y la vulnerabilidad (elementos expuestos) en un espacio geográfico determinado (Lavell, 1996; Cardona, 1996; Wilches, 1998; Mansilla, 2000).

Los asentamientos humanos se construyen y se configuran modificando o transformando la naturaleza En ciertos casos, estos asentamientos se establecen en zonas de riesgo que no pueden ser habilitadas bajo ningún tipo de intervención técnica, económica y ambiental. En este contexto surge la gestión del riesgo como proceso, en el que la sociedad reconoce y valora los riesgos frente a los cuales se encuentra expuesta y, en consecuencia, formula políticas, estrategias, planes e intervenciones con el fin de reducirlo y evitar que surjan nuevos o controlar los ya existentes (Predecan, GTZ, 2006), con prácticas integradas hacia el logro de pautas de desarrollo humano, económico, ambiental y territorial, sostenibles. Esto admite, en principio, distintos niveles de coordinación e intervención, que van desde lo global, integral, sectorial y macro-territorial hasta lo local, comunitario y familiar (CEPREDENAC - PNUD 2003; PDPAE, 2006).

La gestión del riesgo incluye cuatro componentes: prevención, como conjunto de medidas anticipadas para evitar nuevos riesgos; mitigación, como políticas para reducir el riesgo; preparación y atención, orientadas a dar respuesta en casos de desastre, y rehabilitación y reconstrucción, llevadas a cabo post-evento y orientadas al restablecimiento de un territorio luego de sufrir una crisis. Esta gestión del riesgo es abordada de manera correctiva, mediante la reducción del riesgo existente y de manera prospectiva, enfocada a evitar que surjan nuevos riesgos (Predecan, GTZ, 2006; PDPAE, 2006).

Dicha gestión se caracteriza por su relación con el desarrollo; requiere ser abordada como un proceso y no como un producto; está sujeta a la participación de los actores y promueve la creación de estructuras. Una gestión que cobra mayor relevancia en el ámbito local, pues es a esta escala donde el problema se evidencia y se vive con mayor intensidad, en donde los actores 
afectados pueden construir más fácilmente espacios de interlocución y acción frente a la gestión, articulados con procesos de desarrollo y planificación en el contexto de las ciudades (CEPREDENAC - PNUD 2003).

En Bogotá se ha definido un marco normativo y un plan estratégico para abordar la gestión del riesgo, cuyos objetivos giran en torno a la localización, la construcción y la operación segura de asentamientos, también a la inclusión del riesgo en la cultura, a la visibilidad y a la corresponsabilidad frente a los procesos de generación del riesgo, atención integral a las emergencias y resiliencia frente a los desastres (PDPAE, 2006).

Dicho plan define un escenario de gestión del riesgo como aquel que reúne los actores claves de cada uno de los procesos de generación del riesgo para concertar y coordinar la gestión y aumentar la autogestión. Incluye ocho ámbitos de acción, entre los cuales está el de ZONA DE LADERA que corresponde a sectores de periferia urbana al oriente de Bogotá caracterizados por el desarrollo de vivienda ilegal, canteras activas, abandonadas o en recuperación, entre otros (PDPAE, 2006).

La gestión del riesgo incluye el manejo político en el contexto del ordenamiento territorial, a diferencia de la práctica tradicional que se concentraba en la idea de espacializar, por medio de mapas de zonificación, los fenómenos ocurridos sin abordar necesariamente las las directrices políticas tomadas previamente a su ocurrencia (Hewitt, 1996).

En Colombia se trabaja en la incorporación de la gestión del riesgo en la planificación territorial, como una necesidad para la construcción de municipios seguros y sostenibles, con la articulación de medidas estructurales y no estructurales de reducción del riesgo. A su vez, se han rechazado las prácticas de urbanización de zonas "poco aptas" para asentamientos humanos y, por el contrario, se sigue insistiendo en la construcción de políticas que promuevan un orden territorial más armónico con el entorno (MAVDT, 2005); esto es parte de las formas como el estado puede contribuir a mejorar la calidad de vida.

Al respecto, se define por gobernabilidad la capacidad del estado para cumplir sus funciones, ejercer su autoridad y realizar adaptaciones y procesos para mejorar la relación con la sociedad (Whittingham, 2002; Velásquez, 2007), en donde ésta última acepta los productos del sistema político y las decisiones que toman los gobernantes, bajo dos elementos significativos: legitimidad y eficacia del Estado. Por el contrario, crisis fiscal, problemas de autonomía, legitimidad y cohesión de las instituciones, disminución o pérdida de credibilidad por parte de la sociedad en las instituciones del estado, o crisis de gestión administrativa, conducen a la ingobernabilidad (Urdaneta, 2003) y se convierten en un hecho que confronta el 
papel y alcance del ejercicio del poder central.

De otro lado, ha surgido una nueva forma de articulación de los actores públicos y privados en torno a las decisiones públicas conocida como gobernanza, en la cual se deciden, ejecutan y evalúan asuntos de interés público, en donde las instituciones estatales y no estatales, los actores públicos y privados, participan y cooperan en la formulación y aplicación de políticas públicas, en el fortalecimiento del desarrollo sostenible y la democracia (Mayntz, 2001; Whittingham, 2002; Ruano, 2002; Morduchowicz y Arango, 2007), mediante la inclusión de arreglos informales con un objetivo común, dando lugar a una relación de tipo horizontal y concertada entre el Estado y los demás actores (Velásquez, 2007). Para hablar de gobernanza, deben existir unas condiciones principalmente relacionadas con la distribución del poder, en donde hay una condición distinta de la sociedad civil, la disposición y administración de recursos con mayor regulación sobre los mismos, así como la existencia de una sociedad civil fuerte y organizada (Lecay, 2006; El Ágora, 2004).

En la gobernanza moderna existe una relación entre la participación ciudadana, la conformación de redes de organizaciones y el contexto local en el cual se expresan. Un elemento distintivo es el papel de la sociedad civil en las decisiones, lo cual puede ser más efectivo en la escala local (Paredes, 2007), en donde se ejerce el derecho colectivo de manifestar intereses y participar en la formulación y toma de decisiones (Navarro, 2002), lo cual conduce, en algunos casos, a la conformación de redes, que se definen como la interacción de un conjunto de actores interesados en la elaboración de políticas públicas y cuyas características fundamentales se basan en el grado de intensidad que constituye la red, la normalización de la misma, su estandarización y frecuencia de las interacciones (Ruano, 2002), en el marco de la construcción de una política o solución a un problema común.

Por su parte, la participación ciudadana es entendida como la oportunidad individual o colectiva que tienen los ciudadanos de manifestarse frente a un asunto determinado, a través de diversos mecanismos (Navarro, 2004), siendo ésta efectiva a través de información, consulta, co-decisión Y co-gestión; las dos últimas, relacionadas con la gobernanza (Navarro, 2002).

Marc Hufty (2006) desarrolló una propuesta metodológica, con base en la idea de que la gobernanza puede estudiarse a través de la disociación de elementos constitutivos observables, a los cuales el autor llamó categorías: actores, normas, nodos, procesos; estos cuatro, parten tomando como referencia una problemática específica que requiera ser analizada.

Los actores fueron definidos como individuos o grupos de individuos, cuyas acciones conducen a la concertación de 
acuerdos y normas. Las normas corresponden a las reglas del juego, formales o informales, que orientan el comportamiento de los actores y pueden, a su vez, ser modificadas por ellos. En cuanto los nodos o puntos nodales, el autor los identifica como espacios físicos $o$ virtuales, en donde pueden converger actores, o procesos, o normas, y en los cuales pueden surgir nuevos elementos que traigan efectos sobre un proceso o norma en sí. Finalmente, los procesos son concebidos como sucesiones de estados por los que pasan los actores, normas y puntos nodales (Hufty, 2006).

\section{Descripción de la zona de estudio}

Nueva Esperanza es una zona de ocupación y vivienda ilegal que forma parte de la Unidad de Planeación Zonal Diana Turbay (figura 1). Limita por el oriente con el Parque Entre Nubes, al occidente con los barrios San Ignacio y San Martín, al sur con el límite administrativo de las localidades Rafael Uribe y Usme y al norte con el barrio Diana Turbay. Este sector pertenecía, hasta el año 2003, a la localidad de Usme y hacía parte de la UPZ 56-El Danubio pero, por acuerdo del Consejo Distrital No. 117 del 2003, se integró a la UPZ 55- Diana Turbay (Hospital Rafael Uribe Uribe, 2007).

La ocupación del sector empezó aproximadamente en la década de los años 1980, pero sólo hasta 1990 se dio un poblamiento continuo, a partir de la venta de lotes por parte de cuatro invasores del terrenos, quienes sectorizaron la zona y comenzaron a vender. Ocho familias fueron loteando $\mathrm{y}$ vendiendo a muchas personas que llegaron en busca de una opción de tierra para construir y habitar, quienes comenzaron también a acondicionar accesos peatonales a los diferentes sectores de Nueva Esperanza (Hospital Rafael Uribe Uribe, 2007; com. pers. de los habitantes). 


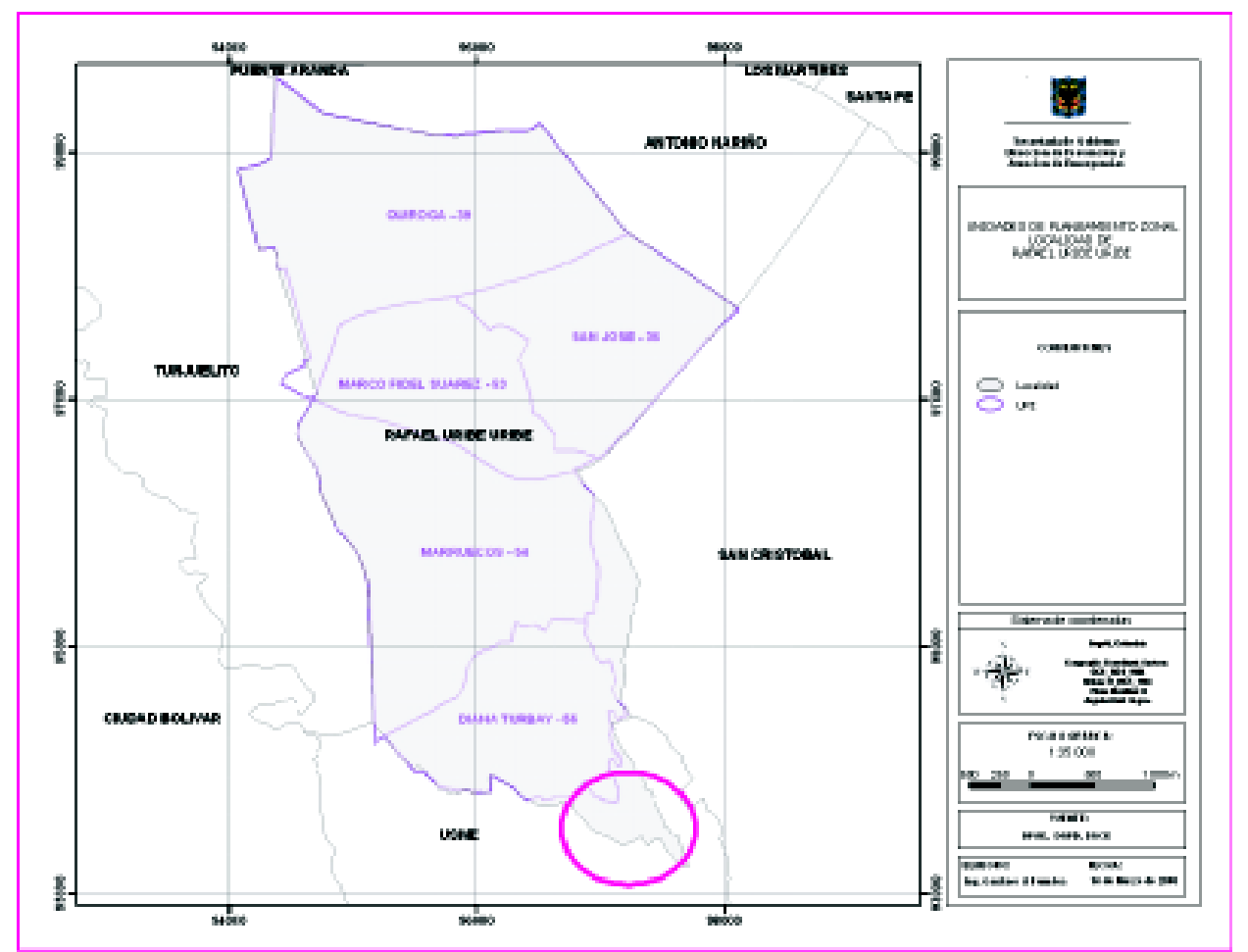

Nueva Esperanza

Figura 1. Localización de Nueva Esperanza según las UPZ de Rafael Uribe Uribe

Fuente: PLGR, 2008

En cuanto el asentamiento ilegal, se ha establecido que el 6\% invadió área de ronda de la quebrada La Guairita, un $1.8 \%$ está invadiendo zona de protección requerida por una línea de transmisión eléctrica y un $57 \%$ ocupó área que pertenece al Parque Ecológico Distrital de Montaña Entrenubes - PEDEN. De acuerdo con los estudios de amenaza realizados en la zona, el terreno es inestable y susceptible a remoción en masa, especialmente en las laderas coluviales, con reptación y flujos de tierra. Esta susceptibilidad también incluye factores asociados con acciones antrópicas tales como deforestación, cortes en la ladera, rellenos, fugas de ductos y proceso de urbanización. (Dpae-Fopae, 2000).

La vulnerabilidad de la población se refleja en las condiciones de pobreza en que habitan, con construcciones en su mayoría improvisadas con latas; no 
cuentan con alcantarillado y, por tanto, deben trasladar el agua en mangueras improvisadas. La economía es informal, las ocupaciones principales son vendedores ambulantes o empleadas del servicio doméstico y, adicionalmente, existen pandillas, violencia intrafamiliar, delincuencia común, reclutamiento de personas para grupos ilegales, reciclaje y expendios de drogas (Secretaría de gobierno, 2006; CVP, 2008).

Entre 2000 y 2004 la remoción en masa provocó diversas emergencias que fueron atendidas puntualmente por las entidades competentes. El 17 de noviembre de 2004 ocurrió una emergencia que superó en su impacto a las precedentes, lo que obligó a la declaratoria de emergencia por parte del Alcalde Mayor, a través del Decreto 383 de noviembre 26 de 2004 y la determinación de elaborar un Plan Integral de Rehabilitación, Reconstrucción y Recuperación post-evento, adoptado mediante Resolución 139 de 2005. Esta determinación probablemente introduciría una manera de abordar la gestión del riesgo más cooperativa y de mayor corresponsabilidad entre diversos actores, comparada con la manera tradicional de la atención que se venía haciendo a la zona hasta antes de dicha emergencia.

\section{M ateriales y M étodos}

Inicialmente, un análisis retrospectivo permitió identificar el proceso de ocupación del sector y las causas y consecuencias del riesgo por remoción en masa. Posteriormente, se aplicó el marco analítico de la gobernanza, propuesto por Hufty (2006), con el fin de conocer la forma de gobernanza que, entre los diferentes actores involucrados, se ha generado para abordar la gestión del riesgo. La figura 2 presenta una síntesis del planteamiento metodológico. 


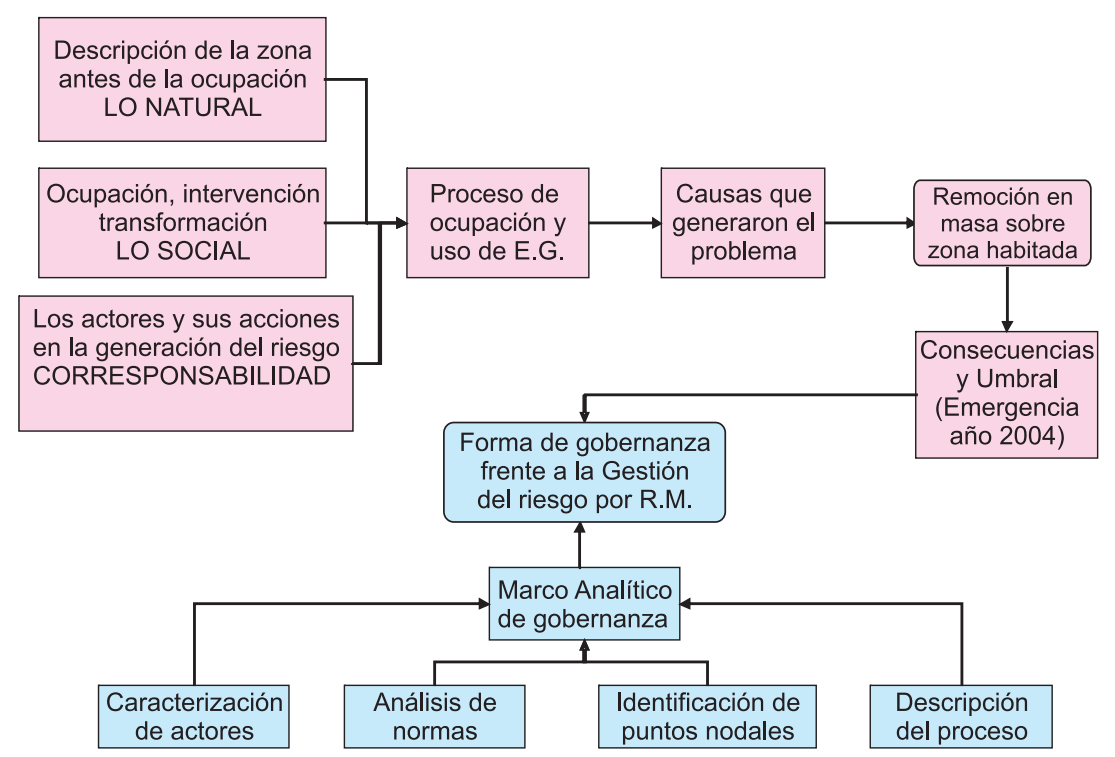

Análisis retrospectivo

Marco Analítoco de Gobernanza

Figura 2. Metodología utilizada durante la investigación Fuente: A. Bedoya, 2008

Un análisis multitemporal de fotografías aéreas permitió analizar los cambios en la organización espacial y distinguir el proceso de ocupación paulatina $\mathrm{y}$, por ende, el cambio en el uso del suelo, contrastando desde la época en donde no existía el asentamiento de Nueva Esperanza, hasta la época en que la zona fue ocupada con viviendas.

Las encuestas fueron aplicadas a la comunidad que aún se encuentra en Nueva Esperanza con el objetivo de obtener en su calidad de "actores" del proceso, su percepción sobre aspectos del trabajo institucional, coordinación entre entidades y la comunidad, resultados de proyectos y acciones, para abordar cuatro variables usadas con frecuencia en otros estudios de gobernanza: participación, rendición de cuentas, eficiencia y equidad. La encuesta se dividió en ocho preguntas asociadas con las cuatro variables.

Se encuestaron personas mayores de edad, según muestra obtenida a partir de la base de datos de "hogares", suministrada por la Caja de Vivienda Popular. El total de población fue de 204 hogares, sobre los cuales se obtuvo una muestra aleatoria que arrojó como cifra representativa un total de 159 hogares para encuestar. Esto se tradujo en un total de 159 personas, en razón de que la encuesta se aplicó a un adulto mayor de edad (preferiblemente cabeza de hogar). 
Fueron entrevistados funcionarios de las entidades distritales involucradas en calidad de "actores" dentro del proceso de Nueva Esperanza. Se escogió uno por cada entidad, buscando que fuera el funcionario más antiguo y con mayor continuidad en la institución. Este criterio de antigüedad, buscaba obtener respuestas fundamentadas en su experiencia o vivencia en terreno sobre cada aspecto en cuestión. Por ejemplo, durante las visitas a los funcionarios en las respectivas entidades, se accedió al Gestor Local del Departamento Administrativo de Acción Comunal, cuya disposición para colaborar en el estudio fue total, pero sólo llevaba dos meses en su cargo y ni siquiera conocía la zona de estudio y mucho menos estaba enterado del proceso adelantado por la entidad que representaba.

Adicionalmente, se diseñó un formulario tipo encuesta para que cada funcionario respondiera, encaminado principalmente a obtener su visión, así como las ventajas y desventajas que pudiera haber observado durante los últimos años, en cuanto la intervención de actores.

Se revisaron los diagnósticos emitidos por la Dirección de Prevención y Atención de Emergencias, DPAE, por medio de los cuales fueron recurrentes los conceptos sobre las condiciones de alto riesgo en la zona. Igualmente, se revisaron Actas del Comité Local de Emergencias y oficios emitidos por la Alcaldía Local de Rafael Uribe Uribe.

Se realizaron seis visitas a la zona: dos en compañía de funcionarios de las entidades involucradas y algunos miembros de la comunidad; las otras cuatro se adelantaron en las jornadas de aplicación de las encuestas.

\section{Resultados}

Esa transformación histórica en la construcción del espacio geográfico, refleja tres aspectos que condujeron a la construcción social del riesgo:

- Cambio en el uso del suelo

- Desarrollo ilegal no planificado

- Ausencia de control por parte del Distrito para evitar los asentamientos ilegales.

\subsection{Cambio en el uso del suelo}

Antes de 1990, predominaban los elementos de la naturaleza, tanto en flora, como en fauna. En los valles del parque, habían pastizales y hacia las laderas helechales que, al parecer, estaban asociados a antiguas quemas. En las cimas aún predominaba la vegetación de subpáramo y matorrales bajos (Corporación Suna Hisca, 2003).

Cuando comienza la ocupación del sector, se empiezan a sacrificar áreas naturales, para adecuar y construir viviendas sin ninguna medida técnica, ni para la intervención del terreno, ni para las obras como tal.

Según se describe en los informes técnicos que evaluaron la amenaza por remoción en masa, los suelos son particularmente sueltos y ni siquiera aptos para 
explotación de materiales tipo cantera.

\subsection{Desarrollo ilegal no planificado}

Para adecuar el terreno, con el fin de construir viviendas, se talaron árboles e intervinieron inadecuadamente las laderas. Para solventar la ausencia de agua, empezaron a transportarla por medio de mangueras elaboradas artesanalmente, con conexiones mal hechas, que ocasionan permanente el derrame o filtración de agua por muchos puntos de la ladera y de la zona plana.

De acuerdo con los estudios técnicos e informes emitidos por las autoridades distritales, con ocasión de la emergencia del 17 de noviembre del 2004, y de la misma manera la comunidad lo reconoce, el principal problema que contribuyó a la emergencia fue la influencia del goteo de agua en diferentes puntos de Nueva Esperanza.

\subsection{Ausencia de control por parte del distrito para evitar los asentamientos ilegales}

Haciendo una mirada retrospectiva, la pregunta principal es: ¿por qué no se tomaron medidas a tiempo para evitar el asentamiento ilegal? Quizás, la respuesta pueda ser tan simple como decir que se tuvieron las normas para evitarlo pero no se aplicaron en rigor. Sin embargo, más allá de una respuesta unilateral a esta pregunta, es necesario una contextualización teniendo en cuenta el papel de los actores, los procesos, las normas y los puntos nodales.

\subsection{M arco analítico de gobernanza}

La tabla 1 ilustra actores principales y secundarios, con el detalle de aquellos que vienen liderando la gestión del riesgo. Estos actores cobran importancia en el marco del Plan de Rehabilitación y Reconstrucción, especialmente por las implicaciones que sus acciones tienen en el reordenamiento territorial. Aquellos actores con impacto importante dentro de la gestión, corresponden a quienes han adelantado proyectos o acciones directas o indirectas, articuladas a la gestión del riesgo. Los actores secundarios están definidos por su papel discreto en el marco del proceso de gestión, lo cual incluye poca presencia de funcionarios en la zona.

Tabla 1. Actores del Proceso

\begin{tabular}{|c|c|c|}
\hline PRINCIPA & LES & SECUNDARIOS \\
\hline Liderando la Gestión del Riesgo & Con impacto importante & Poco presenciales \\
\hline $\begin{array}{l}\text { CVP, DPAE, Secretaría Ambiente, } \\
\text { Control de Vivienda }\end{array}$ & $\begin{array}{l}\text { Hospital RUU, DABS, Alcaldía } \\
\text { Local RUU }\end{array}$ & $\begin{array}{lr}\text { Acción } & \text { comunal, } \\
\text { planeación } & \text { distrital, } \\
\text { ICBF, } & \text { policía } \\
\text { metropolitana de Bogotá }\end{array}$ \\
\hline
\end{tabular}

Fuente: A. Bedoya, 2008

\begin{tabular}{l|l}
\hline 154 & A. Bedoya yJ. Ruiz
\end{tabular} 
La tabla 2 relaciona aquellas con impacto directo en la gestión del riesgo en Nueva Esperanza. Los resultados del estudio indican que, a pesar de existir un amplio marco normativo, su aplicabilidad o cumplimiento no corresponde, en todos los casos, a una experiencia exitosa, especialmente por el desconocimiento de competencias y falta de articulación de las entidades distritales para hacerlas cumplir. Un caso concreto que ilustra esta situación es el control de vivienda en el que, dada la evidencia del caso Nueva Esperanza, se ha demostrado la deficiencia existente en el Distrito Capital para ejercer dicha función.

Tabla 2. Marco Normativo

NORMAS

\begin{tabular}{|c|c|}
\hline MARCO GENERAL & $\begin{array}{l}\text { FORMALIZAN PROCESOS I CON IMPLICACIONES } \\
\text { DIRECTAS EN NUEVA ESPERANZA }\end{array}$ \\
\hline $\begin{array}{lllll}\text { Ley } & 388 & \text { de } & 1997: & \text { Desarrollo }\end{array}$ & DECRETO 383 DE 2004: Declaratoria de emer \\
\hline Decreto 190 de 2004: (POT) & $\begin{array}{l}\text { Resolución } 139 \text { de 2005: Adopción del Plan de } \\
\text { rehabilitación y reconstrucción }\end{array}$ \\
\hline $\begin{array}{l}\text { ACUERDO } 248 \text { DE 2006: Estatuto } \\
\text { General de Protección Ambiental }\end{array}$ & $\begin{array}{l}\text { DECRETO } 546 \text { DE 2007: reglamentación de } \\
\text { comisiones intersectoriales para el control de } \\
\text { vivienda }\end{array}$ \\
\hline $\begin{array}{l}\text { ACUERDO LOCAL } 02 \text { DE 2001: } \\
\text { Plan de desarrollo RUU } 2002 \\
2004 \\
\text { Acuerdo Local No } 003 \text { de 2004: } \\
\text { Plan de desarrollo RUU } 2005 \\
2008\end{array}$ & $\begin{array}{l}\text { DECRETO } 94 \text { DE 2003: Reglamentación del VUR } \\
\text { Complementarios: Convenio interinstitucional SDA- } \\
\text { FOPAE - IDIPRON; Diagnósticos técnicos sobrealto } \\
\text { riesgo }\end{array}$ \\
\hline
\end{tabular}

Nacional

Distrital

Local

Fuente: A. Bedoya, 2008

Según la información analizada e ilustrada en la tabla 3, los espacios de relacionamiento entre actores o nodos fueron periódicos entre el año 2005 y 2006. Durante 2007 y 2008 hubo una desarticulación de estos espacios, total o parcialmente. Esto estaría asociado con el proceso de reasentamiento de la población, especialmente porque los principales líderes comunitarios de la zona entraron entre los primeros beneficiarios a quienes la Caja de Vivienda Popular les otorgó su opción habitacional lejos del sector afectado. Bajo esas características, el reasentamiento de familias provocó un traslado paulatino de personas que anteriormente conformaban redes de vecinos que compartían en común el mismo problema y bajo su interconexión 
de vecindad dialogaban sobre el tema. Al ser trasladados a otros barrios, esa interconexión se pierde y en la medida en que el programa de reasentamiento incorporaba familias y éstas eran trasladadas, más vínculos entre vecinos se rompían, lo que sumado a la pérdida de líderes en el sector, hizo que los nodos antes existentes fueran perdiendo su fuerza y representatividad, aún en espacios interinstitucionales en los cuales podían hacer presencia. Otro hecho significativo, que cobra importancia, es que los espacios creados son utilizados por las entidades y cuentan con muy poca presencia de la comunidad afectada, tal como se evidencia en las listas de asistencia de dichos espacios. Este hecho se ha interrelacionado con los datos arrojados en las cuencuestas, en las cuales existe una parte de la población que manifiesta no creer en las entidades y por ende, consideran poco relevante hacerse presente en espacios donde van a escuchar las mismas explicaciones de las entidades, sin encontrar soluciones reales a sus necesidades. Finalmente, parece que algunos de los nodos tuvieron vigencia con el propósito de difundir o socializar un resultado o una propuesta concreta (sin desconocer la importancia de la socialización) y no parecen haberse creado para llevar a cabo debates o discusiones en pro de los más afectados. El análisis realizado no permite inferir que estos nodos o espacios de relacionamiento hayan producido resultados concretos en términos de compromisos concertados entre la institucionalidad y la sociedad civil.

Tabla 3. Puntos Nodales

\begin{tabular}{|cl|}
\hline NODOS & \\
\hline FORMALES & INFORMALES \\
\hline Mesa de reasentamiento & Espacio permanente en \\
Comité intersectorial de control de vivienda & Nueva Esperanza, a \\
Punto de Atención al Ciudadano - Nueva Esperanza & través de la oficina de \\
Mecanismos de participación Hospital RUU & Secretaría de Ambiente \\
Reuniones interinstitucionales & \\
\hline
\end{tabular}

Fuente: A. Bedoya, 2008

El seguimiento de acciones, la frecuencia de las reuniones o encuentros y la permanencia de un mismo funcionario en cada espacio de encuentro, son aspectos que inciden directamente en el desempeño y aporte de cada nodo a la gestión del riesgo.
En cuanto al análisis de procesos de la problemática de gobernanza del riesgo en Nueva Esperanza, la tabla 4 recoge los más relevantes, en torno de los cuales han girado las actividades contempladas, especialmente aquellas estipuladas en el Plan de Rehabilitación y Reconstrucción para Nueva Esperanza. 
De dichos procesos, es necesario recalcar que el control de nuevas ocupaciones ha sido complicado y difícil de materializar con eficacia en el territorio, entre otros motivos, por la falta de claridad en las competencias institucionales para ejercer dicho control. Cada proceso allí relacionado tiene más un sello de intervención institucional, que de propuestas de construcción colectiva, concertada, en donde la comunidad hubiera tenido una participación activa.

Tabla 4. Proceso identificado en la Gestión del Riesgo en Nueva Esperanza

PROCESOS

\begin{tabular}{|lll|}
\hline DESCRIPCIÓN & RESPONSABLES & RESULTADO \\
\hline $\begin{array}{l}\text { Diagnósticos para determinación } \\
\text { predios en alto riesgo }\end{array}$ & DPAE & $\begin{array}{l}\text { Efectuado } \\
\text { Efectuado fquedan } \\
\text { algunas familias sin } \\
\text { ingresar al proceso) }\end{array}$ \\
Reasentamiento de familias & DPAE - CVP & $\begin{array}{l}\text { Efectuado (en la medida } \\
\text { del desmonte de predios) }\end{array}$ \\
Rehabilitación de la zona & DPAE - SDA & Deficiente \\
Control de nuevas ocupaciones & Lontrol de vivienda - Alcaldía \\
\hline
\end{tabular}

Fuente: A. Bedoya, 2008

A ciertos: cada uno de los señalados en la tabla 5, se ven reflejados directa o indirectamente en la actual organización espacial en Nueva Esperanza. Especialmente, a través del programa de reasentamiento de familias y la recuperación ambiental de las áreas evacuadas, cuya labor ha sido ejecutada por los vigías ambientales mediante convenio interinstitucional. Esto constituye un aporte en materia de uso del suelo.
Desaciertos: Se debe resaltar que al inicio del programa de reasentamiento no existía claridad metodológica para su desarrollo. Esto dificultó y retrasó su ejecución. En 2008, a pesar de que el reasentamiento de la totalidad de habitantes de Nueva Esperanza debía haber llegado a su final, aún existían alrededor de 110 familias que habitan el sector. 
Tabla 5. Aciertos, desaciertos y retos del Proceso de Gestión del Riesgo

RESULTADOS

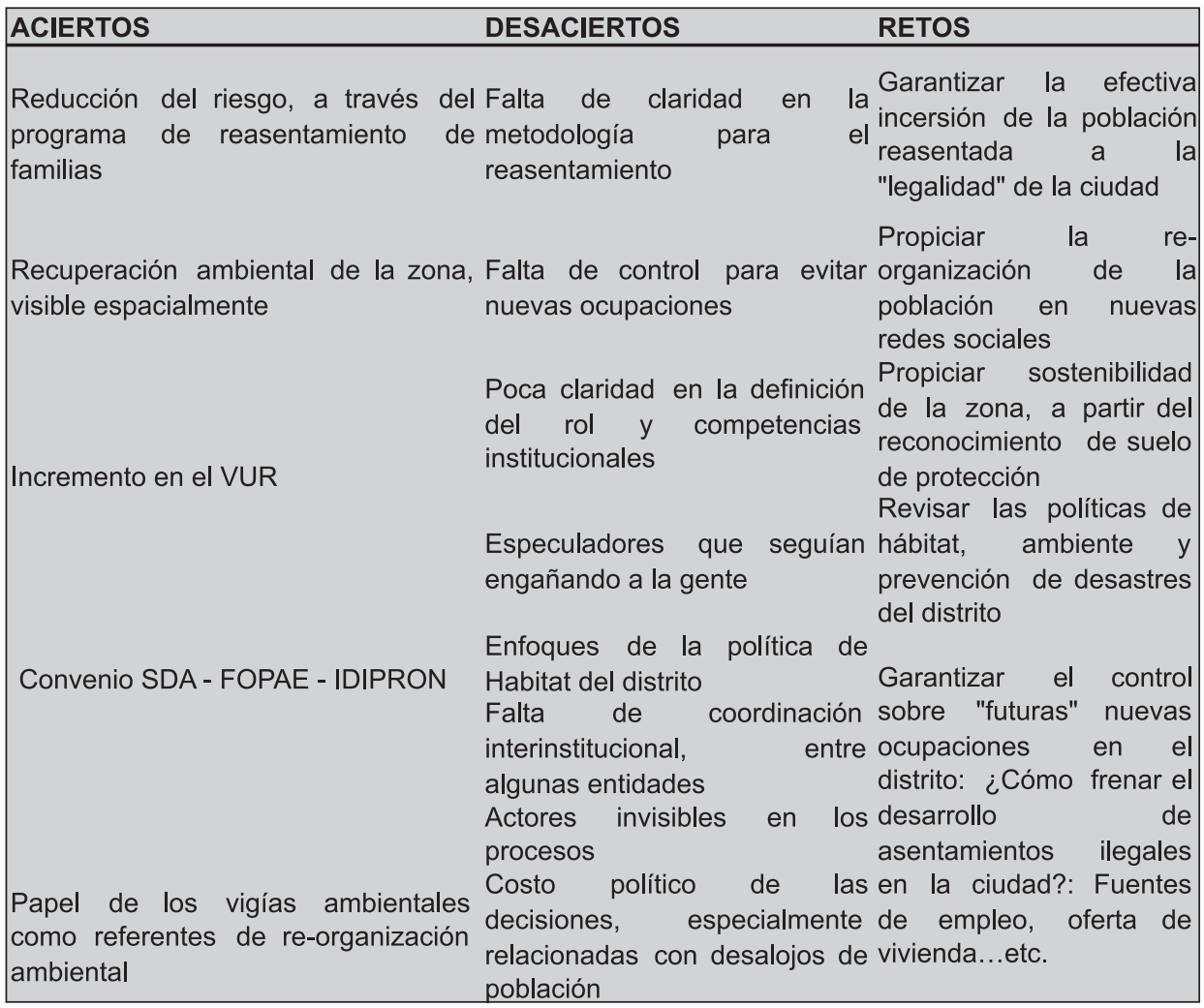

Fuente: A. Bedoya, 2008

Otro aspecto señalado en la tabla 5 es, una vez más, la falta de control sobre nuevas ocupaciones con posterioridad a la declaratoria de emergencia distrital. Este hecho también tiene relación con la poca claridad en las competencias de cada entidad y, en especial, de la cooperación interinstitucional para enfrentar problemas que requieren acciones conjuntas.

Retos: Unos de los mayores retos identificados en esta investigación, está relacionado con la respuesta a la pregunta: ¿cómo garantizar que la historia en Nueva Esperanza no se repita?. Esto se refiere, ni más ni menos, a la sostenibilidad de la ocupación y uso del suelo en el sector a partir de su recuperación ambiental.

\subsection{Discusión}

El análisis retrospectivo del espacio geográfico provee herramientas fundamentales que permiten encontrar 
las causas y comprender los procesos que han incidido en la construcción del riesgo.

Históricamente, en Bogotá ha existido una clara deficiencia en la planificación territorial, con impactos negativos, como la extinción de gran parte de humedales y la localización de un alto número de población en zonas de laderas inestables. Ejemplo claro de ello son numerosos asentamientos y barrios en las localidades de Ciudad Bolívar, Usme y San Cristóbal, donde se presentan una problemática que es una muestra fiel de la convergencia de factores de riesgo en sus espacios geográficos: insuficiencia de control y atención por parte del estado, vulnerabilidad social y económica, así como una trayectoria histórica que ha marcado su actual y progresivo proceso de degradación (Lavell, 1996).

El territorio debe ser un eje conductor de análisis rigurosos de los problemas geográfico-ambientales en el presente, sin desconocer aquello que los teóricos de los riesgos han denominado "la base material de los desastres", entendiendo que, al igual que el espacio, los riesgos son marcadamente una construcción social.

El espacio geográfico, la configuración territorial y los procesos, así como los riesgos y las condiciones de vulnerabilidad, resultan ser un buen motivo para definir integralmente una agenda de trabajo, que incluya la búsqueda de alternativas urgentes y propuestas, de manera prospectiva, al mediano y largo plazo.

En el marco del desarrollo, crecimiento y expansión de centros urbanos es conveniente ahondar en una agenda que desde la geografía contribuya con la generación de nuevo conocimiento y con la conceptualización sobre los riesgos naturales que, como se ha insinuado, parecen incrementarse en vez de reducirse.

De lo observado en el caso de Nueva Esperanza, se puede concluir que Nueva Esperanza es un reflejo de un proceso de organización espacial y de ordenamiento territorial inapropiado, en el cual se evidencia la falta de control por parte del distrito para evitar la ocupación ilegal en la ciudad. Este hecho cuestiona de manera directa la eficiencia del distrito, bien sea para formular políticas más radicales en el control y uso del suelo, o en la aplicabilidad de las mismas a través de sus diferentes entidades.

En Nueva Esperanza, los esfuerzos propuestos por el distrito para llevar a cabo una gestión del riesgo bajo los parámetros de corresponsabilidad, interacción y cooperación entre actores para la gestión del riesgo, sólo se plantearon a partir de la emergencia del 17 de noviembre de 2004. Con anterioridad a esa fecha, la tendencia era "atencionista" ante cada emergencia presentada. Este hecho debe señalar e insistir en abordar el riesgo, el desastre y la gestión correctiva y prospectiva del 
riesgo como elementos ligados a la realidad territorial y la ordenación del territorio, cuya discusión en los espacios de planificación cobre cada vez mayor atención, con el fin de pasar de abordar el tema solo cuando algo superó la capacidad de respuesta y control, para abordarlo como un elemento central que se gesta en la dialéctica sociedad naturaleza, lo cual requiere de atención $\mathrm{y}$ trabajo permanente.

Existe falta de coordinación entre las entidades del distrito. Especialmente, frente a un tema tan delicado como el control sobre nuevas ocupaciones ilegales, en ésta investigación se observaron varias falencias. Una de ellas está documentada con el análisis de cartas que salieron del despacho de la alcaldesa local hacia el comandante de policía del CAI Diana Turbay. En dichas casas se evidencia la falta de coordinación para manejar el tema.

Todos estos elementos: falta de coordinación entre entidades, una comunidad no organizada, falta de eficacia del distrito para ejercer control sobre el territorio y evitar asentamientos ilegales, son aspectos que cuestionan sobre la efectividad y aplicabilidad rigurosa de las políticas, determinaciones y lineamientos que puedan contemplarse en los instrumentos de planificación territorial.

Nueva Esperanza, en cifras contempladas solo a partir de la emergencia del 2004 (no incluye cifras de años anteriores), ha demandado una inversión de \$ 49.761.200.000. En Bogotá hay 9151 predios en zona de alto riesgo, ¿será necesario preparar grandes inversiones para empezar a controlar la situación; o podrán enfocarse mejor las políticas y acciones que le apunten a una construcción correctiva y prospectiva de la Gestión Local del Riesgo, que posibilite actuar a tiempo sin demandar tanto esfuerzo y dinero?

La gobernanza y la gestión del riesgo, como aporte al ordenamiento territorial son, sin duda, un camino por explorar que pueden tener un fin común: propiciar mejores condiciones y calidad de vida para la población, para tener ciudades o centros poblados menos expuestos a riesgos, más preventivos y menos sujetos a la ocurrencia de desastres. 


\section{Literatura citada}

Alcaldía Local Rafael Uribe Uribe. 2008. Plan Local de Gestión del Riesgo. Bogotá, D.C.

Borja, M. 2000. Estado, sociedad y ordenamiento territorial en Colombia. Bogotá, D.C.: Instituto de Estudios Políticos y Relaciones Internacionales de la Universidad Nacional. CEREC serie textos $\mathrm{N}^{\circ} 27$.

Caja de Vivienda Popular. 2005. Marco de política y metodología de reasentamientos de población localizada en zonas de alto riesgo no mitigable, rondas de cuerpos de agua. Bogotá, D.C.

Caja de Vivienda Popular. 2008. Plan de reasentamiento para población localizada en zonas de alto riesgo no mitigable. Zona de Nueva Esperanza. Bogotá, D.C.

Cardona, O. 1996. Manejo ambiental y prevención de desastres: dos temas asociados. LA RED. pp. 57-74. CEPREDENAC - PNUD. 2003. La gestión local del riesgo, nociones y precisiones en torno al concepto y la práctica. Ciudad de Guatemala, Guatemala.

CORPORACIÓN SUNA HISCA 2003., Parque ecológico distrital de montaña Entrenubes. Bogotá, D. C.

Dpae - Fopae. 2000. Estudio de zonificación de riesgos por remoción en masa y recomendaciones de medidas de prevención y mitigación para el caso del barrio de Nueva Esperanza, localidad de Usme, Bogotá. Bogotá, D.C.: Estudio adelantado por la Unión Temporal Geocing Ltda y Bateman Ingeniería.

El Ágora. 2004. Gobernanza y seguridad urbana en América Latina y El Caribe. Realización: Lauub, C. y Bisig, E.. Serie: Aprendiendo de la innovación. Córdoba, Argentina.

García, V. 1996. El estudio histórico de los desastres. En: Historia y desastres en América Latina. pp. 5-20. LA RED.

Gómez, D. 2002. Ordenación territorial. Madrid: Mundi - Prensa en coedición con Agrícola Española, S.A.

Gould, P. 1996. El espacio, el tiempo y el ser humano. Revista Internacional de Ciencias Sociales, $\mathrm{N}^{\mathrm{o}} 150$. www.unesco.org, se tuvo acceso en octubre de 2008.

Harvey, D. 1977. Urbanismo y desigualdad social. México, D.F.: Siglo veintiuno.

Hewitt, K. 1996. Daños ocultos y riesgos encubiertos: haciendo visible el espacio social de Los desastres. En: Desastres, modelo para armar. pp. 23-46. Lima, La Red. 
Hospital Rafael Uribe Uribe. 2007. Por una Esperanza...limpia y saludable. Bogotá. D.C.: Publiscript Impresores.

Hufty, M. 2006. Marco conceptual y analítico de la gobernanza. Documento Congreso Mundial de salud pública. http://www.idrc.ca/uploads/user-S/11556776031Paper_Hufty_Fr.doc, se tuvo acceso en abril de 2008.

Lavell, A. 1996. Degradación ambiental, riesgo y desastre urbano. Problemas y conceptos: hacia la definición de una agenda de investigación. En: Ciudades en riesgo. Degradación ambiental, riesgos urbanos y desastres. pp. 12-42. Lima, LA RED.

Lecay, R. 2006. La gobernanza de los sistemas educativos en América Latina. FLAPE. Foro latinoamericano de políticas educativas. Boletín referencias. $N^{\circ} 20$, Año 3.

Maynz, R. 2001. El estado y la sociedad civil en la gobernanza moderna. Revista del CLAD Reforma y democracia. $\mathrm{N}^{\circ} 21$. Caracas.

Mansilla, E. 2000. Riesgo y Ciudad. LA RED.

Massiris, Á. 2003. Políticas latinoamericanas de ordenamiento territorial: realidades y desafíos. Tesis de doctorado. México, D.F.: Universidad Nacional Autónoma de México. Facultad de Filosofía y Letras. Instituto de Geografía.

Ministerio de Ambiente, Vivienda y Desarrollo Territorial. 2005. Incorporación de la prevención y la reducción de riesgos en los procesos de ordenamiento territorial. Bogotá, D.C.: Guía metodológica 1, Serie Ambiente y Ordenamiento Territorial.

Morcillo, P. 2002. La Planeación en Colombia. Historia, derecho y gestión. Bogotá, D.C.: Ediciones jurídicas Gustavo Ibáñez. Universidad Piloto de Colombia.

Morduchowicz, A y Arango, A. 2007. Gobernabilidad, gobernanza y educación en Argentina. Organización de las Naciones Unidas para la educación, la ciencia y la cultura. Instituto internacional de planeamiento de la educación. Trabajo solicitado por el Programa de las Naciones Unidas para el Desarrollo. http://www.iipebuenosaires.org.ar, se tuvo acceso en abril de 2008 .

Navarro, C. 2002. Gobernanza en el ámbito local. Departamento de ciencia política y de la administración, Universidad Autónoma de Madrid. IX Congreso Internacional del CLAD sobre la reforma del estado y la administración pública. http://www.clad.org.ve, se tuvo acceso en marzo de 2008.

Navarro, J. 2004. Participación ciudadana en la gestión parlamentaria: el concepto de auditoría social. IX Congreso Internacional del CLAD sobre la reforma del estado y la administración pública. Madrid. http://www.clad.org.ve, se tuvo acceso en marzo de 2008 
Ortega, J. 2000. Los horizontes de la geografía teoría de la geografía. Barcelona: Ariel Geografía.

Paredes, J. 2007. Otra democracia: sociedad civil, ciudadanía y gobernanza local. Notas para la discusión. Santiago Polis Universidad Bolivariana. Año/vol. 5 Nº 016. ISSN. 0717-6554.

Plan Distrital de Prevención y Atención de Emergencias. 2006. Decreto 423 de 2006. Dirección de Prevención y Atención de Emergencias. Alcaldía Mayor de Bogotá.

Predecan, PDRS, GTZ. 2006. Incorporación del análisis del riesgo en los procesos de planificación e inversión pública en América Latina y El Caribe. Lima Memoria y resultados del taller internacional.

Ramírez, F. 1996. Elementos conceptuales para el estudio social de los desastres. En: Terremotos en el trópico húmedo. La gestión de los desastres del Alto Mayo, Perú (1990,1992), Limón Costa Rica (1991) y Atrato Medio, Colombia (1992). Maskrey, A. Editor. pp. 20-27. LA RED.

Ruano, J. 2002. La gobernanza como forma de acción pública y como concepto analítico. VII Congreso Internacional del CLAD sobre la reforma del estado y la administración pública Lisboa. http://www.clad.org.ve, se tuvo acceso en marzo de 2008.

Santos, M. 2000. La Naturaleza del Espacio. Barcelona: Ariel S.A.

Secretaría de Gobierno Distrital. 2006. Plan de acción en convivencia y seguridad ciudadana localidad de Rafael Uribe Uribe. Bogotá, D.C.

Urdaneta, A. 2003. Gobernabilidad y participación. El referendo revocatorio en la situación actual de Venezuela. VIII Congreso Internacional del CLAD sobre la reforma del estado y la administración pública. Ciudad de Panamá. http://www.clad.org.ve, se tuvo acceso en julio de 2008.

Velásquez, E. 2007. La Gobernabilidad y la gobernanza de la seguridad ciudadana. Hacia una propuesta operacional. Borrador de Método. Área de Evaluación. www.grupometodo.org, se tuvo acceso en marzo de 2008.

Wilches, G. 1998. Los desastres y el desarrollo. En: Auge, caída y levantada de Felipe Pinillo. pp. 64 - 74. Lima, Red de estudios sociales en prevención de desastres en América Latina.

Whittingham, M. 2002. Aportes de la teoría y la praxis para la nueva gobernanza. VII Congreso Internacional del CLAD sobre la reforma del estado y la administración pública Lisboa http://www.clad.org.ve, se tuvo acceso en abril de 2008. 
Zoido, F. 1998. Geografía y ordenación del territorio. Scripta Vetera, edición electrónica de trabajos publicados sobre Geografía y Ciencias Sociales. Barcelona: reproducido en: Íber, Didáctica de las ciencias sociales. Geografía e historia. Nuevas fronteras de los contenidos geográficos. N 16. pp. 19-31. www.us.es/giest/art_zoido.htm. 1998, se tuvo acceso en julio de 2008.

Fecha de recepción: 12 de diciembre de 2008

Fecha de aprobación: 29 de diciembre de 2008 